\section{International Scientific Journal Theoretical \& Applied Science}

\author{
p-ISSN: 2308-4944 (print) e-ISSN: 2409-0085 (online) \\ Year: $2016 \quad$ Issue: 4 Volume: 36 \\ Published: $30.04 .2016 \quad$ http://T-Science.org
}

Alla Nikolaevna Uglova Associate Professor, Candidate of Pedagogical Science, Assistant professor Department of Physics, State Maritime University Admiral Ushakov, Russia, allauglova@mail.ru

SECTION 21. Pedagogy. Psychology. Innovation in Education.

\title{
DESIGN METHODICAL MAINTENANCE OF SCIENTIFIC DISCIPLINES
}

Abstract: Theoretical bases of designing of methodical maintenance of general scientific disciplines, highlighted the logic of the formation of a training module structure is considered the procedure of formation of a rough basis of activity.the innovative directions of the multi-level educational programs for different categories of students in higher education based on the widespread use of e-learning resources.

Key words: academic discipline, methodological support, training module design, approximate basis of activity.

Language: Russian

Citation: Uglova AN (2016) DESIGN METHODICAL MAINTENANCE OF SCIENTIFIC DISCIPLINES. ISJ Theoretical \& Applied Science, 04 (36): 138-140.

Soi: http://s-o-i.org/1.1/TAS-04-36-22 Doi: crossef http://dx.doi.org/10.15863/TAS.2016.04.36.22

\section{УДК 372.851}

\section{ПРОЕКТИРОВАНИЕ МЕТОДИЧЕСКОГО ОБЕСПЕЧЕНИЯ ОБЩЕНАУЧНОЙ ДИСЦИПЛИНЫ}

Аннотация: Представлены теоретические основы проектирования методического обеспечения общенаучной дисциплины, выделена логика формирования структуры учебного модуля, рассмотрена прочедура формирования ориентировочной основы деятельности.

Ключевые слова: учебная дисциилина, методическое обеспечение, учебный модуль, проектирование, ориентировочная основа деятельности.

\begin{abstract}
Методическое обеспечение учебной дисциплины включает совокупность материальных (учебно-методическая литература, ТСО и т.д.) и интеллектуальных (принципы, методы, методики и т.д.) средств, обеспечивающих проектирование и реализацию образовательного процесса. При этом методическое обеспечение может быть двухуровневым и включать: методическое обеспечение проектирования учебной дисциплины, определяющее процедуры формирования учебной дисциплины (выбор изучаемых объектов, определение последовательности их изучения, выбор организационных форм и определение последовательности учебных занятий и т.д.); методическое обеспечение учебной дисциплины,
\end{abstract}

определяющее специфику реализации самого образовательного процесса (частные методики проведения учебных занятий, учебные пособия, методические рекомендации обучаемым, различного рода учебные задания, методики решения задач и т.д.).

Логика формирования методического обеспечения цикла отражена на рис.1. Типовая структура модуля УЭ для всех этапов обучения определяет как компонентный состав модуля, так и его функциональные характеристики (последовательность изучения компонентов модуля; методы и организационные формы обучения, способы формирования ООД и т.д.) [4]. 


\begin{tabular}{|c|c|c|c|c|c|c|}
\hline Impact Factor: & $\begin{array}{l}\text { ISRA (India) } \\
\text { ISI (Dubai, UAE } \\
\text { GIF (Australia) } \\
\text { JIF }\end{array}$ & $\begin{array}{l}=1.344 \\
=0.829 \\
=0.564 \\
=1.500\end{array}$ & $\begin{array}{l}\text { SIS (USA) } \\
\text { PИНЦ (Russia) } \\
\text { ESJI (KZ) } \\
\text { SJIF (Morocco) }\end{array}$ & $\begin{array}{l}=0.912 \\
=0.234 \\
=1.042 \\
=2.031\end{array}$ & $\begin{array}{l}\text { ICV (Poland) } \\
\text { PIF (India) } \\
\text { IBI (India) }\end{array}$ & $\begin{array}{l}=6.630 \\
=1.940 \\
=4.260\end{array}$ \\
\hline
\end{tabular}

Типовая структура модуля УЭ общенаучной дисциплины

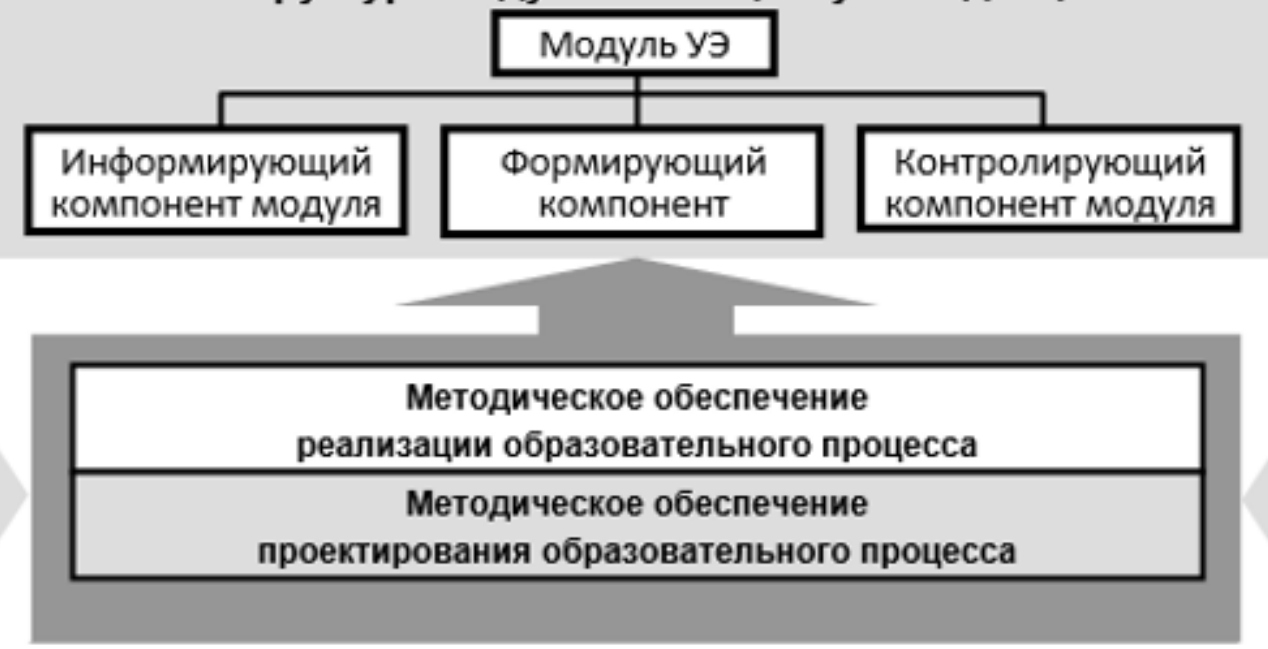

Рисунок 1- Логика формирования методического обеспечения.

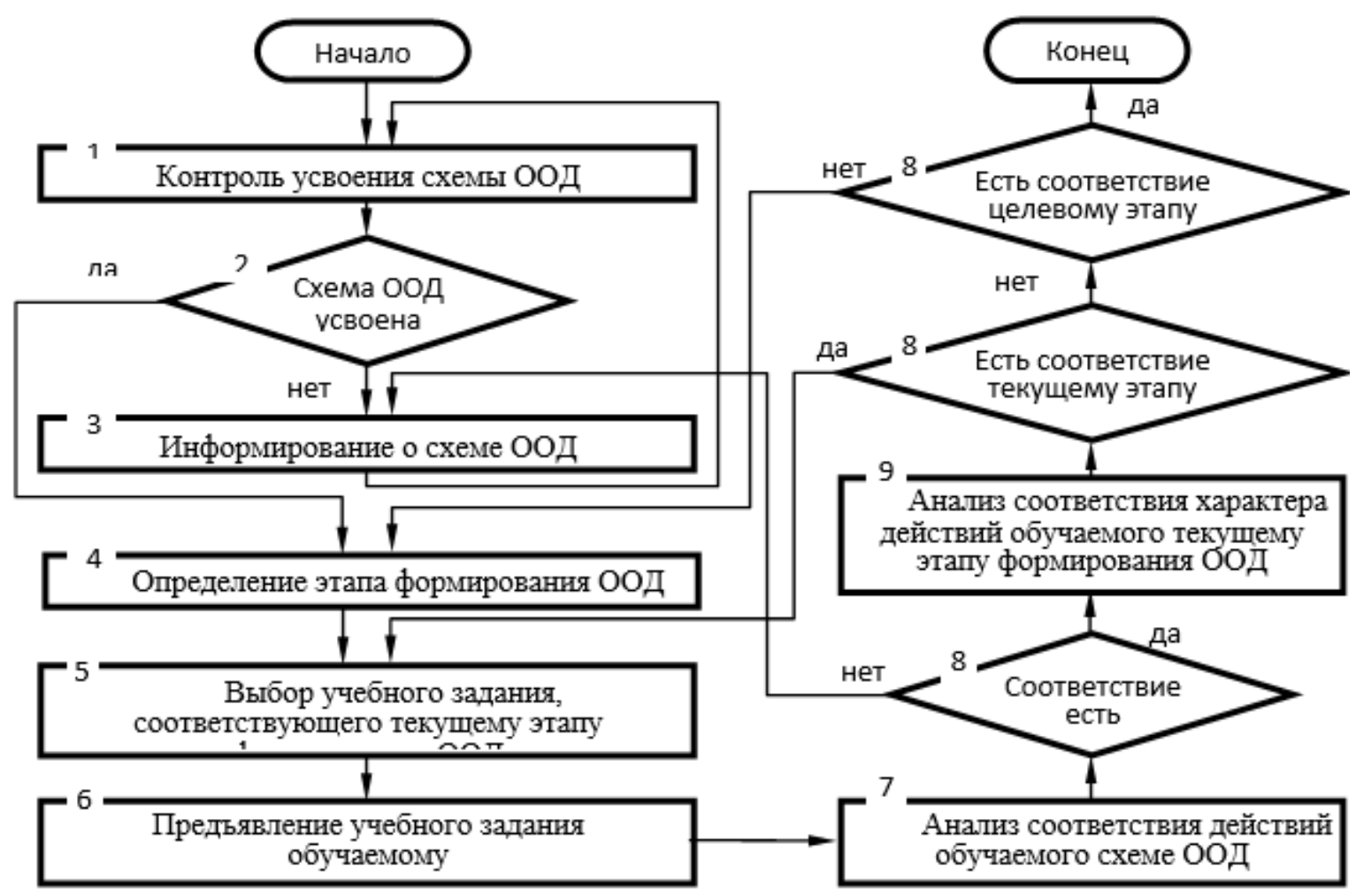

Рисунок 2 - Процедура управления процессом формирования ООД, адекватная индивидуальным потребностям обучаемых.

Цель первого этапа усвоения модуля УЭ ограничена предъявлением обучаемым схемы ООД. На втором этапе в процессе учебной деятельности происходит формирование этой схемы. На третьем этапе осуществляется итоговый контроль формирования ООД и используется только контролирующий компонент модуля УЭ.

На основе анализа данных теории интериоризации была разработана [2] процедура формирования ООД, адекватная потребностям обучаемого (см. рис. 2). Цель разработки методического обеспечения формирующих видов занятий (практические занятия, лабораторные занятия и т.д.) состояла в обеспечении реализации этой процедуры.

Проведенная на основе указанных выше требований разработка методического обеспечения позволила осуществить единый подход к формированию структуры и функций
ISPC Global Science,

Lancaster, USA
THOMSON REUTERS

Indexed in Thomson Reuters 
методических пособий, как к практическим занятиям, так и к лабораторным занятиям.

В процессе практических и лабораторных занятий студент должен понять и усвоить не только определенные теоретические закономерности, но и модель формируемой деятельности, уяснить ее строение и способ организации. Поэтому необходимо, чтобы содержание методических пособий отражало не только знание о предмете, но и знания о строении деятельности, ее состава, функциональной структуры, способах выполнения деятельности $[1,3]$.

Усвоение содержания учебного материала происходит в процессе познавательной деятельности по его применению. Организация познавательной деятельности курсантов на лабораторно-практических занятиях должна способствовать развитию личности, формированию высокой культуры умственного труда, интереса к учению, потребности в самостоятельном творческом поиске.

\section{References:}

1. AN Uglova (2014) The organization of educational process on the basis of innovative technologies. Materials eleventh regional scientific-technical conference: Problems of operation of water transport and training in the south of Russia.- Novorossiysk: RIO State University. adm. Ushakov, 2014.

2. Balyaeva SA (1999) Theoretical Foundations fundamentalization general scientific training in the system of higher technical education: Author. Dis Dr. ped.nauk.-M., 1999. - 32 p.

3. Reshetova ZA (1985) Psychological bases of vocational training. - M .: Izd. Univ., 1985

4. AN Uglova (2005) Methodical maintenance fundamentalization general scientific training of the Navy reserve officers in a technical college (for example, "Physics" discipline) .- Dis .... kand.ped.nauk. 13.00.08.--SPb 2005

5. Balyaeva SA, Uglova AN (2012) Improving the efficiency of general scientific training of the maritime industry specialists on the basis of innovative teaching technologies // scientific research humanitarian issues. Issue 5 Pyatigorsk. 2012.

6. Pechnikov AN, Vetrov YA (2002) Design and application of computer technology training. Part 1. The concept of automated training systems and modeling of business. Kn.2. - St.
Petersburg: Baltic State Technical University, 2002.

7. Mishchik SA (2014) Pedagogometrika and mathematical modeling educational activity. Materialy Mezhdunarodnoy nauchnoy konferenctsii "Modern mathematics in science" - 30.06.2014. ISJ Theoretical \&Applied Science 6(14): 54-56 Caracas, Venezuela. doi: http://dx.doi.org/10.15863/TAS.2014.06.14.10.

8. Balyaeva SA (2002) Formation of systems thinking as a condition of fundamental nature and the professionalization of assimilated knowledge. - In the book $\therefore$ Formation of systems thinking in education. - M : UNITY DANA 2002.

9. Balyaeva SA, Uglova AN, Baloyan ON (2015) Theoretical aspects of the design of didactic maintenance of general scientific training in the Maritime University / Proc .: Education and era (current scientific paradigm) .- 5. BookMoscow: Science: Inform; Voronezh SGMP 2015

10. Balyaeva SA, AN Uglova (2014) Theoretical basis of pedagogical innovations // Bulletin of the State Maritime University named after Admiral FF Ushakov. Exploitation. Safety and economics of water transport. - 2014. - №2 (7). 